\title{
Interaction des résidus d'anthelminthiques dans le lait de vache avec la flore bactérienne et Penicillium roqueforti
}

\author{
C Longin-Sauvageon ${ }^{1}$, JC Beguin ${ }^{2}$, M Florent ${ }^{3}$ \\ 1 INRA, Laboratoire de biochimie, Ecole nationale vétérinaire de Lyon, 1 avenue Bourgelat, B.P. 83, \\ 69280 Marcy-l'Etoile; \\ 2 Laboratoire Rhône-Mérieux, 4 chemin du Calquet, 31057 Toulouse; \\ ${ }^{3}$ Laboratoire CESAR, 1, rue Hermann-Frenkel 69364 Lyon Cedex 07, France
}

(Reçu le 19 avril 1989; accepté le 27 juillet 1989)

\begin{abstract}
Résumé - Une série de 9 anthelminthiques destinés aux ruminants a été testée chez la vache en vue de la mise en évidence d'un éventuel pouvoir inhibiteur des résidus éliminés dans le lait, sur les technologies de fabrication des fromages à maturation bactérienne ou fongique. Aucun d'entre eux, ou leurs métabolites n'a été reconnu comme étant doué d'activité antibactérienne. En revanche, une activité antifongique a été mise en évidence in vitro pour certains composés à l'égard du Penicillium, mais ce phénomène ne semble pas responsable d'anomalies de fabrication.
\end{abstract}

anthelminthique / résidu / lait / antibactérien / antifongique

Summary - Interaction of residues of anthelminthics in cow's milk with bacteria and Penicillium roqueforti. The inhibitory properties of milk residues on cheese technology (bacterial and fungic maturation) have been studied in 9 anthelminthics which are largely used in ruminants. None of them, or their metabolites, is antibacterial. On the contrary, some of them, belonging to the benzimidazole series are antifungic by in vitro tests but do not seem to produce anomalies in cheese fabrication.

antheminthic / milk / residue / antibacterial / antifungic

\section{INTRODUCTION}

L'utilisation d'anthelminthiques en médecine vétérinaire, peut se traduire par la présence dans le lait de résidus responsables d'effets indésirables sur sa qualité, avec répercussion éventuelle sur les fabrications fromagères mettant en jeu des fermentations bactériennes ou fongiques. 
Ainsi, le thiabendazole, dont les résidus dans le lait de chèvres perturbent la fabrication de efromage bleu" (Toussaint et al, 1976), est reconnu comme puissant inhibiteur des Penicillium (Davidse et Flach, 1978). En revanche, ces résidus n'ont aucune action sur la flore bactérienne du lait de brebis (Pisanu, 1965). D'autre part, le bithionol sulfoxyde, à des concentrations élevées, diminue l'acidification du lait et exerce une légère inhibition des ferments lactiques utilisés dans les tests de détection des antibiotiques (De Laistre Banting et Paviot, 1986).

L'objectif du présent travail est de déterminer quels sont les anthelminthiques et leurs métabolites éliminés dans le lait de vache susceptibles d'inhiber le développement des flores bactérienne et fongique, et modifiant éventuellement les fabrications fromagères. Cette étude a été réalisée en mettant en cuuvre les étapes suivantes : détermination des niveaux résiduels dans le lait après traitement, aptitude à la fabrication de fromages, établissement des éventuelles concentrations minimales inhibitrices et étude de la liaison de métabolites actifs aux protéines du lait.

\section{MATÉRIEL ET MÉTHODES}

\section{Anthelminthiques étudiés}

Les composés retenus pour la présente expérimentation sont des anthelminthiques commerciaux couramment utilisés en thérapeutique vétérinaire :

- l'oxyclozanide $(3,3$ ',5,5',6-pentachloro2,2'-dihydrobenzanilide, OCZ, Zanil N.D. Coopers);

- le netobimin ( $N$-methoxycarbonyl- $N-N(2-$ nitro-5-propylthiophenyl)- $N^{\prime \prime}$-(2-sulfoethyl) guanidine, NTB, Hapadex N.D. Schering);

- l'albendazole (methyl(5-(propylthio)-1H benzimidazole-2-yl)carbamate, ABZ, Valbazen N.D. SKF); Ltd);

- le ricobendazole (SO·ABZ, Robert Young

- le thiabenzole (2-(4'-thiazolyl) benzimidazole, TBZ, Thibenzole N.D. Merck);

- le lévamisole (2,3,5,6-tetrahydro-6-phenylimidazo(2,2-6)thiazole, LVM, Bionem N.D. Coopers);

- le fébantel ( $\mathrm{N}$-2-(2,3-bis-(methoxycarbonyl)-guanidine)-5-(phenylthio)-phenyl-2-methoxyacetamide, FBT, Rintal N.D. Bayer);

- le fenbendazole ( $N$-phenylthio-5, benzimidazolyl-2, carbamate de méthyle, FBZ, Panacur N.D. Distrivet).

- l'oxfendazole (SO•FBZ, Synanthic N.D. Rhône-Mérieux);

- le thiophanate éthyl (diethyl(1,2-phénylène bis iminocarbonothioyl) bis carbamate, Strongynate N.D. Véprol);

- le lobendazole (benzimidazole carbamate d'éthyl, BCE, SKF).

A ces composés ont été joints pour les tests in vitro:

- le luxabendazole (5-(4-fluorophenylsulfonyloxy) benzimidazole-2-carbamic acid methyl ester, LXZ, Fluxacur N.D. Distrivet).

- le nitroxynil (4-cyano-2-iodo-6-nitrophenol, NXN, Dovenix N.D. Rhône-Mérieux);

- le triclabendazole (6-chloro-5-(2,3-dichlorophenoxy)-2-methyl thiobenzimidazole.

Outre les composés chimiquement purs, ont été soumis aux tests de laboratoire, certains métabolites synthétiques correspondants : le triclabendazole sulfoxyde (SO-TCZ) et triclabendazole sulfone (SO2TCZ), l'albendazole sulfone (SO2ABZ), le 5-hydroxy-thiabendazole $(\mathrm{OH} \cdot \mathrm{TBZ})$ et le fenbendazole sulfone (SO2FBZ).

\section{Traitements et prélèvements}

Parmi les anthelminthiques énumérés, 9 d'entre eux (tableau I) ont fait l'objet d'une administration orale à des vaches laitières (race française frisonne pie noire), à des doses 1,5 fois supérieures à la dose thérapeutique normale. Les laits correspondant à 3 traites consécutives dont le rang est fonction de la pharmacocinétique chez la vache, ont été collectés et stockés immédiatement à $+4{ }^{\circ} \mathrm{C}$ jusqu'à acheminement aux laboratoires et usines de fabrication. 


\section{Détermination des niveaux résiduels}

Ils ont été déterminés par des méthodes empruntées à la littérature, ou mises au point pour la présente expérimentation : extractions organiques suivies d'analyse par chromatographie haute performance ou par chromatographie gazeuse (Simkins et al, 1976; Delatour et al, 1983; Zeil, 1988).

\section{Essais de microfabrications}

Des microfabrications fromagères par maturation par flore bactérienne (yaourt, fromage frais et emmental) et fongique ("bleu"), ont été réalisées par des industriels selon les bonnes pratiques industrielles, après traitement par le LVM, TBZ, OCZ, FBT, FBZ et SO•FBZ. En cours de fabrication, un certain nombre de paramètres ont été périodiquement évalués pour contrôler l'évolution de la fabrication : acidité Dornic, $\mathrm{pH}$, pénétration Stevens, numération des germes totaux et bactérioscopie, texture et goût.

\section{Essais d'inhibition bactérienne}

Les techniques utilisées sont celles officiellement mises en œuvre pour la détection des antibiotiques dans le lait (François, 1981). Elles comportent : une méthode d'acidification en milieu liquide utilisant Streptococcus thermophilus comme germe-test, et 3 tests de diffusion en gélose avec Bacillus stearothermophilus, B. subtilis et $B$. megaterium. Ces 4 tests ont été réalisés dans les conditions suivantes :

$-2 \mathrm{ml}$ de lait cru sont pasteurisés et ensemencés par le germe-test;

$-2 \mathrm{ml}$ de lait cru sont pasteurisés, additionnés d'éthanol $(40 \mu \mathrm{l})$ et ensemencés;

$-2 \mathrm{ml}$ de lait cru sont pasteurisés, supplémentés et ensemencés;

$-2 \mathrm{ml}$ de lait cru sont supplémentés, pasteurisés et ensemencés.

Les supplémentations en principes actifs et métabolites ont été réalisées sous un volume constant de $40 \mu \mathrm{l}$ d'éthanol selon une gamme de concentration finale de 0 à $5 \mu \mathrm{g} / \mathrm{ml}$.

\section{Essais d'inhibition fongique}

\section{Matériel}

Il est constitué des éléments suivants : d'un Autodiluteur III Dynatech, de Microplaques 96 puits (Dynatech MA 1703 A), d'un Lecteur de microplaques MR 610, de Gélose : milieu solide Sabouraud modifié (Bio-Mérieux), et de spores de Penicillium roqueforti : 2 souches ont été utilisées parallèlement: PB 16 et PRB 18 (suspension de travail à $5 \times 10^{2} \mathrm{UFC} / \mathrm{ml}$ ).

\section{Méthode}

La solution mère du principe actif et/ou des métabolites (solution éthanolique à $50 \mu \mathrm{g} / \mathrm{ml}$ ) a été déposée dans les 6 premières cupules, les 2 autres servant de témoin sont additionnées d'eau distillée et d'éthanol. Après dilution de deux en deux par l'autodiluteur (concentrations finales dans le milieu de 0,006 à $6,25 \mu \mathrm{g} / \mathrm{ml}$ ), on ajoute $175 \mu \mathrm{l}$ de milieu de Sabouraud, ensemencé à raison de $10 \%$ avec la suspension de spores de Penicillium. Les microplaques ont été placées à l'étuve à $25^{\circ} \mathrm{C}$ pendant 7 jours. La lecture est alors réalisée par mesure néphélométrique.

\section{Etude de la liaison aux protéines par dialyse}

La liaison du FBZ aux protéines lactiques a été déterminée par dialyse à l'équilibre (Scholtan, 1962), avec un système DIANORM (Diachema A.G., Switzerland; membrane Spectra Por 3). Le lait témoin $(1 \mathrm{ml})$ supplémenté en $\mathrm{FBZ}$ selon différentes concentrations $(0,5-1,0-2,0$ et $5,0 \mu \mathrm{g} /$ $\mathrm{ml}$ ), a été soumis à dialyse pendant $6 \mathrm{~h}$ à $37^{\circ} \mathrm{C}$ contre $1 \mathrm{ml}$ de tampon phosphate $0,01 \mathrm{M} \mathrm{pH}$ 7,4 , identiquement supplémenté. A l'équilibre, les contenus de chaque compartiment ont été récupérés et soumis à extraction organique ( $\mathrm{De}$ latour et al, 1983), puis la concentration en FBZ des extraits a été déterminée par chromatographie liquide à haute performance. Des dialyses de lait supplémenté simultanément, en FBZ et ses deux principaux métabolites SO.FBZ et SO2FBZ, ont été réalisées dans les mêmes conditions. 


\section{RÉSULTATS}

\section{Niveaux résiduels}

La nature et le niveau des résidus dans le lait de vache après traitement oral par 9 anthelminthiques, figurent dans le tableau I.

\section{Essais de microfabrications}

Lors des essais de microfabrications fromagères par maturation par flore bactérienne et fongique, à partir des échan- tillons de lait de vaches traitées, aucune modification n'a été observée par rapport aux essais témoins au vu des critères retenus (examens bactériologiques chimiques et organoleptiques).

\section{Tests d'inhibition bactérienne}

Pour tous les laits expérimentaux et témoins supplémentés en anthelminthiques et leurs métabolites dans la gamme de concentration comprise entre 0 et $5 \mu \mathrm{g} / \mathrm{ml}$, aucune anomalie n'a été observée pour les tests d'acidification et de diffusion en gélose.

Tableau I. Teneurs résiduelles maximales dans le lait de vache après administration orale. Maximal residual content of anthelminthics in bovine milk after oral administration.

\begin{tabular}{|c|c|c|c|}
\hline Principe actif & Dose (mg/kg) & Métabolites & Concentration $(\mu \mathrm{g} / \mathrm{ml})$ \\
\hline Lévamisole (LVM) & 11,25 & LVM & 2,06 \\
\hline Oxyclozanide (OCZ) & 15 & OCZ & 0,002 \\
\hline \multirow[t]{2}{*}{ Thiabendazole (TBZ) } & 40 & TBZ & 0,008 \\
\hline & & $\mathrm{OH} \cdot \mathrm{TBZ}$ & 0,54 \\
\hline Thiophanate & 40 & BCE & 0,44 \\
\hline \multirow[t]{4}{*}{ Nétobimin (NTB) ${ }^{a}$} & 12,5 & NTB & 0,74 \\
\hline & & $A B Z$ & 0,00 \\
\hline & & $S O \cdot A B Z$ & 0,80 \\
\hline & & SO2ABZ & 0,96 \\
\hline \multirow[t]{3}{*}{ Albendazole (ABZ) } & 7,5 & $A B Z$ & 0,00 \\
\hline & & $S O \cdot A B Z$ & 0,80 \\
\hline & & SO2ABZ & 0,96 \\
\hline \multirow[t]{4}{*}{ Fébantel (FBT) } & 11,25 & FBT & 0,00 \\
\hline & & & 0,06 \\
\hline & & SO•FBZ & 0,39 \\
\hline & & SO2FBZ & 0,12 \\
\hline \multirow{3}{*}{ Oxfendazole (SO•FBZ) } & 6,78 & FBZ & 0,19 \\
\hline & & $S O \cdot F B Z$ & 0,39 \\
\hline & & SO2FBZ & 0,12 \\
\hline \multirow{3}{*}{ Fenbendazole (FBZ) } & 11,25 & FBZ & 0,15 \\
\hline & & $\mathrm{SO} \cdot \mathrm{FBZ}$ & 0,27 \\
\hline & & SO2FBZ & 0,11 \\
\hline
\end{tabular}

a Administration par voie sous-cutanée.

a Subcutaneous administration. 


\section{Tests d'inhibition fongique}

Les résultats obtenus n'ont pas montré de différence significative entre les 2 souches de Penicillium testées. La Concentration Minimale Inhibitrice (CMI) des différents anthelminthiques testés, en milieu additionné ou non de lait, figure dans le tableau II. Parmi tous les composés explorés, seuls le lobendazole, l'albendazole, le thiabendazole, le luxabendazole et le fenbendazole présentent une $\mathrm{CMI}$ inférieure à $6,25 \mu \mathrm{g} / \mathrm{ml}$. Pour l'albendazole et le tenbendazole, cette valeur est multipliée par 2 et 10 respectivement, selon que le milieu d'incubation contienne ou non du lait.

\section{Liaison protéique}

Elle a été étudiée uniquement pour le FBZ retrouvé après administration de FBZ, SO.FBZ et SO2FBZ et dont la CMI varie selon la présence ou non de lait dans le milieu. Pour les 4 concentrations de FBZ dans le lait $(0,5-1,0-2,0$ et $5,0 \mu \mathrm{g} / \mathrm{ml})$, les pourcentages de liaison protéique sont les suivants : $96,35 \pm 0,28-96,78 \pm 1,15-$ $96,55 \pm 0,05$ et $69,75 \pm 0,03 \%$. II en résulte que dans le lait de vache, aux faibles concentrations, le FBZ se trouve essentiellement lié aux protéines. Ces valeurs ne sont pas modifiées lors de dialyses de lait en présence de $\mathrm{FBZ}, \mathrm{SO} \cdot \mathrm{FBZ}$ et SO2FBZ.

Tableau II. CMI $(\mu \mathrm{g} / \mathrm{ml})$ des anthelminthiques et de leurs métabolites vis-à-vis de Penicillium roqueforti. $\mathrm{CMI}(\mu \mathrm{g} / \mathrm{ml})$ of anthelminthics and their metabolites towards Penicilium roqueforti.

\begin{tabular}{|c|c|c|}
\hline Principe actif ou métabolite & Milieu + éthanol & Milieu + lait \\
\hline Lévamisole & a & a \\
\hline Oxyclozanide & $a$ & $\mathbf{a}$ \\
\hline $\begin{array}{l}\text { Thiabendazole } \\
\mathrm{OH} \cdot \mathrm{TBZ}\end{array}$ & $\begin{array}{c}0,78 \\
a\end{array}$ & $\begin{array}{c}0,78 \\
a\end{array}$ \\
\hline $\begin{array}{l}\text { Thiophanate d'éthyle } \\
\text { BCE }\end{array}$ & $\begin{array}{l}1,56 \\
0,19\end{array}$ & $\begin{array}{l}1,56 \\
0,19\end{array}$ \\
\hline $\begin{array}{l}\text { Nétobimin } \\
\text { ABZ } \\
S O \cdot A B Z \\
S O 2 A B Z\end{array}$ & $\begin{array}{c}a \\
0,39 \\
a \\
a\end{array}$ & $\begin{array}{l}a \\
0,78 \\
a \\
a\end{array}$ \\
\hline $\begin{array}{l}\text { Fébantel } \\
\text { FBZ } \\
\text { SO·FBZ } \\
\text { SO2FBZ }\end{array}$ & $\begin{array}{c}a \\
0,039 \\
a \\
a\end{array}$ & $\begin{array}{c}a \\
0,39 \\
a \\
a\end{array}$ \\
\hline $\begin{array}{l}\text { Triclabendazole } \\
\text { SO·TCZ } \\
\text { SO2TCZ }\end{array}$ & $\begin{array}{l}a \\
a \\
a\end{array}$ & $\begin{array}{l}\mathrm{a} \\
\mathrm{a} \\
\mathrm{a}\end{array}$ \\
\hline Nitroxynil & a & a \\
\hline Luxabendazole & 1,5 & 1,5 \\
\hline
\end{tabular}




\section{DISCUSSION}

\section{Activité antibactérienne}

Les tests antibactériens mis en œuvre, habituellement utilisés pour la détection des antibiotiques dans le lait, semblent valables pour les anthelminthiques, en raison de la cohérence existant entre ces derniers et les essais de microfabrication. En mettant en parallèle les niveaux résiduels dans le lait après administration orale, et les tests in vitro d'activité antibactérienne, i) apparaît qu'aucun anthelminthique ou métabolite correspondant, ne peut être responsable de phénomène d'inhibition dans les processus de caséification. Ces conclusions peuvent s'appliquer au nitroxynil dont l'élimination dans le lait est de l'ordre de $1,0 \mu \mathrm{g} / \mathrm{ml}$ (Takeshita et al, 1980), mais doivent cependant être confirmées pour le triclabendazole, et le luxabendazole, dont on ne connaît pas les cinétiques d'élimination dans le lait.

Ces résultats sont enfin compatibles avec ceux obtenus lors des essais de microfabrications à partir de lait de vaches traitées.

\section{Activité antifongique}

Pour les anthelminthiques et leurs métabolites correspondants qui ne présentent pas d'activité antifongique in vitro leur administration ne peut être responsable d'incident de fabrication par inhibition de Penicillium, les niveaux résiduels dans le lait étant dans tous les cas inférieurs à $6,25 \mu \mathrm{g} / \mathrm{ml}$. Cette éventualité est rencontrée pour LVM, OCZ, TCZ et NXN.

Au contraire, pour les autres composés un effet inhibiteur in vitro, a été mis en évidence pour la molécule parentale ou l'un de ses métabolites :
- le thiabendazole, puissant inhibiteur des Penicillium (Davidse et Flach, 1978) est connu de longue date comme inhibiteur de la fabrication de fromage "bleu" à partir de lait de brebis; il en va de même chez la chèvre (Toussaint et al, 1976). La présente étude indique que ce phénomène n'est pas retrouvé à partir du lait de vache. Le TBZ, doué d'activité antifongique (CMI dans le lait : $0,8 \mu \mathrm{g} / \mathrm{ml}$ ), est métabolisé en composé phénolique $\mathrm{OH} \cdot \mathrm{TBZ}$, totalement dépourvu de cette activité. La différence de comportement entre la brebis et la vache repose sur le fait que, chez la brebis, le TBZ n'est que faiblement converti en OH•TBZ (Weir et Bogan, 1985) alors que chez la vache, l'oxydation du TBZ est très abondante, au point que le TBZ inchangé est indétectable dans la circulation sanguine (Prichard et al, 1985). II en résulte que chez la brebis les résidus dans le lait sont essentiellement constitués de TBZ, alors que chez la vache, ceux-ci sont exclusivement représentés par $\mathrm{OH} \cdot \mathrm{TBZ}$ non antifongique. Une situation intermédiaire est représentée par la chèvre (Tocco et al, 1965). La différence métabolique entre la brebis et la vache rend compte du fait que le TBZ est préjudiciable à la fabrication de fromage bleu à partir de lait de brebis, mais non à partir de lait de vache.

- l'ABZ, qui présente une $\mathrm{CMI}$ dans le lait de $0,78 \mu \mathrm{g} / \mathrm{ml}$, ne peut être à l'origine de phénomène d'inhibition fongique. En effet, après son administration chez la vache il est rapidement converti en $S O \cdot A B Z$ et SO2ABZ inactifs qui sont les seuls métabolites circulant dans le plasma (Fetterer et al, 1982; Prichard et al, 1985; Delatour et al, 1987). Pour le netobimin (NTB), une prodrogue de I'ABZ, ainsi que pour le ricobendazole ( $\mathrm{SO} \cdot \mathrm{ABZ}$ ), ils constituent également les seuls résidus mis en évidence (Delatour et al, 1986; Thiberghien et Bogan, 1987); 
- en ce qui concerne le fenbendazole, ce composé est retrouvé dans le lait après administration de FBT, FBZ et de SO FBZ (Delatour et Euzéby, 1983; Delatour et al, 1983), à des doses toujours inférieures à la CMI dans le lait : $0,39 \mu \mathrm{g} / \mathrm{ml}$. Ce fait interdit toute activité antifongique. On remarque par ailleurs que la $\mathrm{CMI}$ dans le lait est 10 fois supérieure à celle déterminée dans le milieu de Sabouraud, probablement en raison de la forte liaison $(96 \%)$ aux protéines lactiques. L'activité inhibitrice du métabolite FBZ s'exercerait probablement si la teneur résiduelle était suffisante pour saturer les sites de fixation sur les protéines et permettre ainsi l'existence d'une fraction libre capable d'exercer l'effet inhibiteur. Cette éventualité ne pourrait se rencontrer que lors d'un fort surdosage accidentel ( $x$ à 8) en FBT, FBZ ou SO•FBZ, et reste à confirmer in vitro par l'étude de l'interaction du FBZ sur Penicillium roqueforti en présence de caséine.

En revanche, après administration de thiophanate, le niveau résiduel du métabolite actif, le BCE, s'élève à $0,44 \mu \mathrm{g} / \mathrm{ml}$, soit une valeur sensiblement supérieure à la CMI : $0,19 \mu \mathrm{g} / \mathrm{ml}$. Quant au luxabendazole (CMI : $1,5 \mu \mathrm{g} / \mathrm{ml}$ ), non encore commercialisé chez la vache, sa cinétique d'élimination dans le lait n'est pas connue.

Sur l'ensemble des anthelminthiques et des métabolites testés, seuls ceux présentant une activité antifongique, font partie de la classe des benzimidazoles, dont le mécanisme d'action est en partie basé sur l'inhibition de la polymérisation de la tubuline en microtubules (Friedman et Platzer, 1978; Barrowman et al, 1984; Lacey et Prichard, 1986). L'activité antifongique mise en évidence chez les levures et les Penicillium (Davidse et Flach, 1977, 1978), est également basée sur l'inhibition de la polymérisation de la tubuline. Certains anthelminthiques vétérinaires possèderaient donc théoriquement la propriété d'inhiber le processus de maturation de la flore fongique, si après traitement ces composés se retrouvent dans le lait à une concentration supérieure ou égale à la CMI.

\section{CONCLUSION}

La présence dans le lait de vache, de résidus des anthelminthiques retenus pour la présente expérimentation, ne semble pas affecter les tests bactériens et ne saurait être incriminée dans les accidents de fabrication de fromages par maturation bactérienne. Concernant l'activité antiPenicillium, ces anthelminthiques sont, selon les cas, formellement mis hors de cause pour des raisons métaboliques ou éventuellement incriminés lors de forts surdosages accidentels hors des posologies préconisées.

\section{REMERCIEMENTS}

Nous remercions les établissements Rousset (Andrézieux-Bouthéon), F. Bourdin (Echalas) et I'I.T.G. (Rioz) pour leur participation à ce travail. Nous remercions également les laboratoires qui nous ont fourni les composés : Merck, CibaGeiby, Rhône-Mérieux, Coopers, Bayer, Distrivet et S.K.F.

\section{RÉFÉRENCES}

Barrowman MM, Marriner DE, Bogan JA (1984) The binding and subsequent inhibition of tubulin polymerisation in Ascaris suum (in vitro) by benzimidazole anthelmintics. Biochem Pharmacol 33, 3 037-3 040

Davidse LC, Flach W (1977) Differential binding of methyl benzimidazol-2-yl carbamate to fungal tubulin as a mechanism of resistance to this antimitotic agent in mutant strains of Aspergillus nidulans. J Cell Biol 172, 174-193 
Davidse LC, Flach W (1978) Interaction of thiabendazole with fungal tubulin. Biochim Biophys Acta 543, 82-90

De Laistre Banting A, Paviot F (1986) Evaluation des effets inhibiteurs des résidus de bithionol sulfoxyde dans le lait sur les ferments lactiques. Rec Méd Vét 162, 1 005-1 008

Delatour P, Cure MC, Benoit E, Garnier F (1986) Netobimin preliminary investigations on metabolism and pharmacology. $J$ Vet Pharmacol Ther 9, 230-234

Delatour P, Euzéby J (1983) Communautés structurale, métabolique et anthelminthique entre fébantel, fenbendazole et oxfendazole. Point Vét 15, 63-67

Delatour P, Garnier F, Benoit E (1983) Kinetics of four metabolites of febantel in cow's milk. Vet Res Commun 6, 37-42

Delatour P, Gyurik RL, Benoit E, Garnier F (1987) Pharmakinetics of albendazole administered by an intraruminal pulse release electronic device in cattle. Res Vet Sci 43 , 284-286

Fetterer R, Rew RS, Knight R (1982) Comparative efficacy of albendazole against Fasciola hepatica in sheep and calves : Relationship to serum drug metabolite levels. Vet Parasitol 11, 309-316

François A (1981) Instructions pour la détection des antibiotiques et des sulfamides dans les laits livrés par les producteurs. Rev Lait Fr 395, 7-11

Friedman PA, Platzer EG (1978) Interaction of anthelmintic benzimidazoles and benzimidazole derivatives with bovine brain tubulin. Biochim Biophys Acta 544, 605-614

Lacey E, Prichard RK (1986) Interactions of benzimidazoles (BZ) with tubulin from $B Z$ sensitive and $\mathrm{BZ}$-resistant isolated of Haemonchus contortus. Mol Biochem Parasitol $19,171-181$
Pisanu S (1965) Sulla azione del tiabendazole nei processi di fermentazione e caseificazione del latte. Latte 39, 519-521

Prichard RK, Hennessy DR, Steel JW, Lacey E (1985) Metabolite concentrations in plasma following treatment of cattle with five anthelmintics. Res Vet Sci 39, 173-178

Scholtan W (1962) Uber die Bindung der Langzeitsulfonamide and die Serumeiweisskörper. Makromol Chem 54, 24-59

Simkins KL, Smith JE, Eggert RG (1976) Excretion of levamisole in milk from cows treated with various formulations. J Dairy Sci 59, $1440-1443$

Takeshita Y, Kishi T, Seki M, Fujiama K, Otsuka G, Ahiko K (1980) Analysis of nitroxynil (fasciolicide) in milk and dairy products. Milchwischenschaft 35, 133-135

Tiberghien MP, Bogan JA (1987) Albendazole and albendazole sulfoxyde : a comparison of bioavailability in cattle and sheep. Commun Congrès WAAP, Montréal, 12-15 août

Tocco DJ, Egerton JR, Bowers W, Christensen VW, Rosenblum C (1965) Absorption, metabolism and elimination of thiabendazole in farm animals and method for its estimation in biological materials. J Pharmacol Exp Ther 149, 263-271

Toussaint G, Raynaud JP, Tailland J, Portmann A (1976) Perturbation éventuelle de la fabrication, de l'apparence et du goût des fromages, après administration aux vaches laitières d'anthelminthiques des familles benzimidazole ou tetrahydropyrimidine. Rev Med Vet 127, 259-288

Weir AJ, Bogan JA (1985) Thiabendazole and 5 -hydroxythiabendazole in the plasma of sheep. J Vet Pharmacol Ther 8, 413-414

Zeil JM (1988) Contribution à l'étude de l'incidence des résidus de thiabendazole sur les technologies laitières. Thèse Doct Vét $n^{\circ}$ 105, Lyon 